\title{
WALTHER 66, 15.
}

Dass von der nur in C überlieferten Waltherstrophe 66, 13-20

Swie liep si mir von herzen sî, sô mac ich doch vil wol erlîden

15 daz ich ir sî zem besten bî:

ich daiff ir werben dâ niht nîden

u.s.w. die zeile 15 verderbt ist, đürfte feststehen, ebenșo aber auch, dass eine völlig befriedigende heilung der stelle noch nicht gefunden ist. Von älteren vermutungen führt Wilmanns,

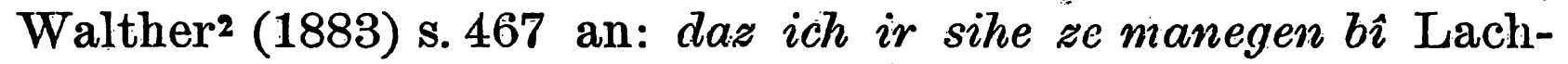

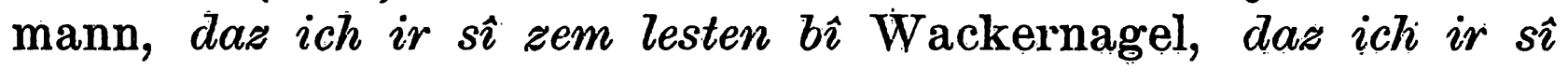
$z e n$ besten bîn Simrock. Von diesen hat keine in die neueren ausgaben eingang gefunden, und mit recht, denn keine gibt einen brauchbaren gedanken (die von Simrock dürfte übrigens den meisten lesern wol ebenso unverständlich sein; wie mir; vgl. s. 205, anm.). Sonst ist mir nur noch der zweifelnde vorschlag von Scherer, Anz. fda. 10, 310: daz ich ir sihe goste bî bekannt geworden, der Lachmanns sike (das als indicativ hier an sich schon etwas befremdlich ist) aufnimmt, mit geste aber näher an dem besten der hs. bleibt. Deswegen mag wol Wilmanns diese lesung in die zweite auflage seiner kleinen textausgabe (Halle 1905, no. 63, 19) aufgenommen haben. Paul lehnt sie dagegen stillschweigends mit ab (Walther ${ }^{3}$, Halle 1905, no.32,19), und ich kann das nur billigen. Denn ich sehe nicht, was die geste hier sollen. Zumal bei Walthers ganz prägnanter gebrauchsweise des` wortes gast (s. die stellen bei Horning s. 102) könnte der satz ja nur heissen 'dass ihr fremde ins haus gekommen sind', und das steht wenigstens für mich nicht in einem greifbaren bezug zu den getrogenen und dem rïemic man von 66, $19 \mathrm{f}$., zu denen doch irgend eine brücke hinüberführen muss (weiteres dazu unten s. 205 f.). So mag es denn gestattet sein, einem fünften emendationsversuch 
raum zu geben, der mir vor jahren einmal eingefallen ist, und für den äusserlich vielleicht mit spricht, dass er sich (wie ich erst jetzt beim niederschreiben dieser zeilen bemerke) der allgemeinen gedankenrichtung nach mit einer auffassung berührt, der Simrock in den späteren ausgaben seiner übersetzung ausdruck verliehen hat.

Der grundgedanke der strophe ist ja klärlich in $66,17 \mathrm{f}$. ausgesprochen: Walther fürchtet keine concurrenz, die ihm bei der geliebten schaden könnte, und braucht sie nicht zu fürchten, wie er uns selbstbewusst versichert. Liegt es da nicht nahe zu vermuten, in der verderbten zeile habe ursprünglich etwas gestanden, was den begriff jener concurrenz näher definierte, und zwar nach der steigernden seite hin: 'keine concurrenz, wenn sie auch noch so gefährlich aussieht', oder 'keine concurrenz, und wenn ich auch mit den besten wetteifern muss'? Etwas ähnliches hat, wie angedeutet, offenbar schon Simrock herausgefühlt, wenn er (no.137 der 6. ausgabe) übersetzt: 'So mag ich doch nun wol erleiden, Steht auch dem. besten zutritt frei.'1) Das wäre denn mhd. vermutlich

daz ich ie sî den besten bî,

d. h. 'ich bin ganz damit zufrieden, mich immer nur den besten bewerbern zuzugesellen: auch von diesen wird ja keiner mir eintrag tun können.' Damit gewinnt auch das ir von z. 16

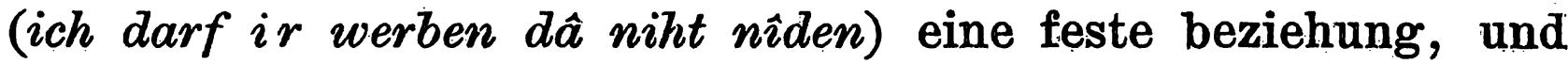
der gedanke wie der vorgeschlagene ausdruck hat eine stütze an 86, 2:

Frouwe, enlât iuch niht verdriezen mîner rede, ob si geviuege sî.

86,1 möhte ichs wider iuch geniezen, sô wær ich den besten gerne bî.

Denn auch da sagt ja Walther, dass er nur mit den besten wetteifern wolle, wenn die dame ihm das zum guten anzurechnen bereit sei (über verwante gedanken s. Wilmanns, Leben Walthers s. 183 nebst den zugehörigen anmerkungen). -

Hart bleibt freilich auch bei dieser auffassung der übergang auf 66, 19 f., denn man wird schwerlich annehmen dürfen, dass die getrogenen und rüemigen den besten gleichzusetzen

1) Etwas ähnliches mag Simrock wol auch mit seinem $d a z$ ich ir sî zen besten $b \hat{\imath}$ gemeint haben: aber diese worte geben das nicht her. 
seien, von denen Walther eben gesprochen hat. Die rüiemigen speciell sind ja offenbar die eitlen praller (vgl. namentlich 41, 16. 25), dann aber die getrogenen auch gewiss diejenigen, die sich leerer hoffnung hingegeben haben, ohne die grundlage inneren wertes, der sie zu einer hoffinung berechtigt (zum ausdruck vgl. mich entriege ein wân 120,37, auch 116, 7; auch an Hartmanns kreuzlied MF. 218, 21 f. mag man mutatis mutandis erinnern): beide aber hätte Walther, der so oft den gegensatz zwischen dem edlen und unedlen werber betont, gewiss nicht den besten zugerechnet, deren mitwerben er sich siegesgewiss gefallen lassen will. Innerhalb unserer strophe finden also die beiden schlusszeilen wol keine ausreichende erklärung. Aber die ganze strophe steht ja so wie so ohne äussern anschluss da (das hat Scherer 2.a.o. ganz riehtig betont, und auch Wilmanns hat die strophe danach in der textansgabe von 1905 wider von den beiden vorhergehenden abgetrennt), and ihr inhalt ist wol auch nicht so geschlossen und abgerundet, dass man sie sich gerne von vornherein so isoliert entstanden dächte, wie sie in der überlieferung dasteht. Ich möchte also vermuten, dass ihr ursprünglich noch mindestens eine andere strophe vorausgegangen sei, auf deren inhalt Walther mit den schlusszeilen 66, $19 \mathrm{f}$ zurückgegriffen habe. Was im einzelnen darin gestanden haben möge, lässt sich natürlich nicht sagen, aber man möchte fast meinen, eine energische absage an alle unedlen werber, aus dem munde der dame (eventuell durch den mund Walthers) sei doch dabei gewesen. Waren dadurch die unedlen von weiterem werben ausgeschlossen, so konnte Walther recht wol fortfahren, dass er auch den mitbewerb der besten nicht fürchte: denn niemand werde seine dame wankend machen können: 'das < aber > freut mich, dass jene betrogenen [die sich eitler hoffnung hingegeben haben] nun erfahren, was sie getäuscht hat [nämlich ihr eigner unwert], und [formell parataktisch anknüpfend, dem sinne nach aber begründend, ‘denn'] schon lange hat mich's verdrossen (mirst ... alee lanc), dass prahler zu ihr zutritt fanden'.1) -

2) Die ubersetzung der letzten zeile ist natiurlich ungenau, aber sie muss es sein, weil sich bei Walther offenbar zwei gedanken gekreuzt haben, nümlich 'mich hat verdrossen, dass prabler zu ibr zutritt gefunden haben' und 'mich verdriesst's, wenn' ein prahler je (iemer, auf die zu- 
Wenn die oben empfohlene conjectur das richtige trifft, so hat sie, um auch das nicht zu übergehen, zugleich noch den vorteil, dass sie sich auch formell dem sprachmelodischen typus des gedichts gut einfügt, was man weder von dem überlieferten text, noch von den andern erwähnten vorschlägen sagen kann. In den vierhebigen versen ${ }^{1}$ ) der drei dem tone nach zusammengehörigen strophen bilden nämlich die hebungen nach niederdeutscher intonation eine (übrigens bei Walther ausserordentlich beliebte) gleichmässig aufsteigende, nach hochdeutscher gleichmässig absteigende tonreihe (also niederdeutsch $\cdot \bullet^{-}{ }^{-}$, hochdeutsch $\bullet^{-} \cdot{ }^{-}$, beidemal mit nur kleinen tonschritten), und so auch bei der lesung daz ich ie sî den besten $b \hat{\imath}$. Bei den andern leseweisen aber wird, wie die probe leicht zeigen kann, die sonst glatte reihe irgendwie gebrochen, am auffälligsten bei der von Scherer vorgeschlagenen daz ich ir sihe geste bî. Bei ihr springt das wort geste nach niederdeutscher intonation übermässig in die höhe, und $b \hat{\imath}$ fällt dann wider nach unten ab, und umgekehrt bei hochdeutscher intonation (also niederdeutsch . • • . , hochdeutsch ${ }^{\bullet} \bullet^{\bullet}{ }^{\bullet}$; ausserdem verschiebt sich bei ihr das niveau der ganzen zeile gegen das der übrigen (nach oben bei niederdeutscher, nach unten bei hochdeutscher intonation).

Man wird allerdings dieser auffassung der sprachmelodischen form unserer strophen und überhaupt der forderung melodischer constanz vielleicht die eingangszeilen 65,33

In einem zwîvellîchen wân was ich gesezzen, und gedâhte

u.s.w. entgegenzuhalten geneigt sein, denn hier hat die erste zeile ja unzweifelhaft auch einen bruch (niederdeutsches schema . $\bullet^{-}$., hochdeutsches $\bullet^{\bullet} .{ }^{-}$), nicht eine glatte ton-

kunft deutend) bei ihr zutritt findet' (sc. wie das nach den gesellschaftlichen verhältnissen nicht zu umgehen ist). - Wilmanns' umschreibung: 'es freut mich, wenn die betrogenen liebhaber sie recht kennen lernen, und doch dauert es allzu lang, dass sie mit ihnen verkehrt' kann ich mit den textworten nicht recht in einklang bringen. Auch bei dieser auffassung bleibt übrigens das iemer ein stein des anstosses. Ueber die annahme einer gedankenkreuzung wird man also wol auf keinen fall hinwegkommen.

1) Gleichviel ob mit stumpfem oder klingendem ausgang: auf die längeren verse braucht hier nicht eingegangen zu werden. 
reihe. Dem gegenüber wird es aber wol genügen, auf Lachmanns anmerkung zu verweisen: 'der dativus wân ist unrichtig: soll man lesen Uf einen zwîvellîchen wân?' (vgl. auch Wilmanns zur stelle und s. 29 f.). Der melodische anstoss trifft also auch hier wider mit einem anstoss anderer art zusammen, und so wird man nun nicht mehr zweifeln dürfen, dass der dativ hier wirklich 'falsch' ist und durch einen acc. ersetzt werden muss (einerlei wie man über die frage des eventuellen $\hat{u} f$ denkt). Stellt man diesen acc. ein, so erscheint sofort auch die ungebrochene tonreihe wider, die ich als für unsere strophen charakteristisch und verbindlich ansehe.

Im vorbeigehen sei dann noch angemerkt, dass auch in 66, 14 sô mac ich doch $\langle$ vil $>$ wol erlî̀en die melodischen verhältnisse gebieterisch die von Lachmann vorgeschlagene. ergänzüng (vil) fordern. Ohne sie fällt die zeile (um von sonstigem abzusehen) wider aus dem niveau des übrigen heraus.

LEIPZIG, 19. november 1908 .

E. SIEVERS.

\section{LITERATUR.}

(Verzeichnis bei der redaction eingegangener schriften).

Brandstetter, Joseph teopold, Die Rigi und der Pilatus, zwei grenzsteine zwischen Helvetien und Rhätien (Sep.-abdr. aus dem Geschichtsfreund bd. LXIII). Stans [1909]. - $42 \mathrm{~s}$.

Brockstedt, Gustav, Das altfranzösische Siegfriedslied. Eine reconstruction. Mit einem schlusswort: zur geschichte der Siegfriedssage. Kiel, R. Cordes, 1908. - XIII, $178 \mathrm{~s}$.

v. Grienberger, Theodor, Das Hildebrandslied (Sitzungsber. d. Wiener Akad. phil.-hist. cl. 158. band, 6. abh.). Wien, Hölder 1908. - $109 \mathrm{~s}$.

Hugo von Trimberg, Der Renner, hg. von Gustav Ehrismann. Band II (Bibliothek des Literar. vereins in Stuttgart 248). Tübingen 1909. - $315 \mathrm{~s}$.

Kauffmann, Friedrich, Zur textgeschichte des opus imperfectum in Matthaeum (Festschrift d. univ. Kiel). Kiel, Lipsius \& Tischer, 1909. - 49 s.

Kiuge, Friedrich, Etymologisches wörterbuch der deutschen sprache. 7. verbesserte und vermehrte auflage. 1. lieferung A-Fohlen. Strassburg, Trübner, 1909. - $144 \mathrm{~s}$.

Meier, John, Werden und leben des volksepos. Rede, geh. d. 15. nov. 1907 am jahresfeste der univ. Basel. Halle, Max Niemeyer, 1909. - 54 s. Paul, Hermann, Prinzipien der sprachgeschichte. 4. auflage. Halle, Max Niemeyer, 1909. - X $\mathrm{X}, 428 \mathrm{~s}$.

Vogt, Friedrich, Der bedeutungswandel des wortes edel. Rede beim antritt des rectorats gehalten am 18. october 1908. Marburg, Elwert, 1908. $-36 \mathrm{~s}$. 